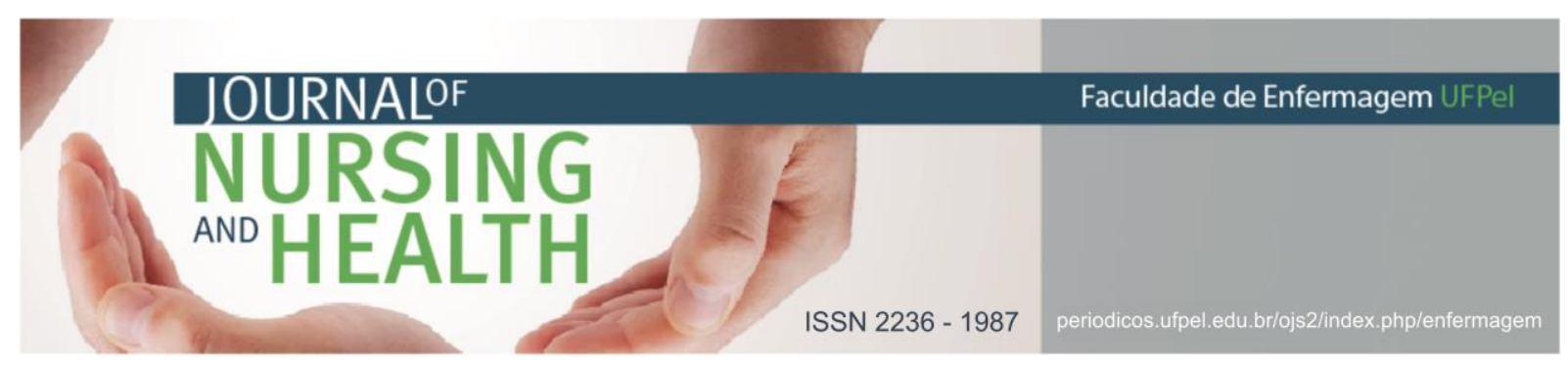

ARTIGO ORIGINAL

\title{
Infecção de sítio cirúrgico em revascularizações do miocárdio: estudo retrospectivo
}

\section{Surgical site infection in myocardial revascularization: a retrospective study}

\section{Infección de sitio quirúrgico en revascularización del miocardio: estudio retrospectivo}

Santos, Heberton Polegato dos¹; Ribeiro, Ariela Cristina²; Lanza, Vinícius Encenha3 ${ }^{3}$ Montandon, Diego Santiago4; Godoy, Simone de ${ }^{5}$

Como citar este artigo: Santos HP, Ribeiro AC, Lanza VE, Montandon DS, Godoy S. Infecção de sítio cirúrgico em revascularizações do miocárdio: estudo retrospectivo. J nurs health. 2018;8(3):e188308

\section{RESUMO}

Objetivo: analisar a incidência de infecção do sítio cirúrgico em revascularizações do miocárdio. Métodos: estudo quantitativo com 314 prontuários de pacientes submetidos às revascularizações no período de 2014 a 2016, do serviço de cirurgia cardíaca. Foram avaliadas as relações de variáveis operatórias com o aparecimento de infecção de sítio cirúrgico para determinar os fatores significativos. Para a coleta dos dados utilizou-se as fichas de circulação extracorpórea e para a análise as definições estabelecidas pela Agência Nacional de Vigilância Sanitária. Resultados: 66,2\% eram do sexo masculino; $62,1 \%$, brancos; $47,3 \%$, hipertensos; $22,8 \%$, diabéticos e $91,7 \%$, cirurgias eletivas. Evidenciou-se que febre, edema local em 24 horas, dor, necrose em lesão operatória e (re)abordagem cirúrgica são os principais fatores para incidência de infecções relacionada ao tempo médio de circulação extracorpórea e de cirurgia. Conclusão: a infecção de sítio cirúrgico em revascularizações está relacionada com o tempo de cirurgia e de circulação extracorpórea.

Descritores: Circulação extracorpórea; Cirurgia torácica; Enfermagem; Infecção hospitalar.

\begin{abstract}
Objective: to analyze the incidence of surgical site infection in myocardial revascularizations. Methods: a quantitative study with 314 medical records of patients submitted to revascularization in the period from 2014 to 2016, of the cardiac surgery service. The relationships of operative variables with the appearance of surgical site infection were evaluated to determine the significant factors. Data collection was done using the cardiopulmonary bypass records and for the analysis the definitions established by the National Sanitary Surveillance Agency. Results: 66,2\% were male; $62,1 \%$ white; $47,3 \%$ hypertensive and $22,8 \%$ diabetic; $91.7 \%$ were elective surgeries. It was evidenced that fever, local edema in 24 hours, pain, necrosis in operative lesion and surgical intervention are

1 Enfermeiro. Universidade do Oeste Paulista (UNOESTE). E-mail: htompolegato@hotmail.com http://orcid.org/0000-0001-6286-753X

2 Enfermeira. Universidade do Oeste Paulista (UNOESTE). E-mail: arielacr89@gmail.com http://orcid.org/00000003-2747-4479

3 Enfermeiro. Universidade do Oeste Paulista (UNOESTE). E-mail: vinicius_lanza@hotmail.com http: / /orcid.org/0000-0002-7652-8093

4 Enfermeiro. Mestre em Enfermagem Fundamental. Universidade do Oeste Paulista (UNOESTE). E-mail: diegosmontandon@gmail.com http://orcid.org/0000-0002-1999-3941

5 Enfermeira. Doutora em Ciências. Universidade de São Paulo (USP). E-mail: sig@eerp.usp.br http://orcid.org/0000-0003-0020-7645
\end{abstract}




\section{JOURNALOF

the main factors for the incidence of infections related to the mean time of extracorporeal circulation and surgery. Conclusion: the surgical site infection in revascularizations is related to the time of surgery and extracorporeal circulation.

Descriptors: Extracorporeal circulation; Thoracic surgery; Nursing; Cross infection.

\section{RESUMEN}

Objetivo: analizar la incidencia de infección del sitio quirúrgico en revascularizaciones del miocardio. Métodos: estudio cuantitativo con 314 prontuarios de pacientes sometidos a revascularizaciones en el período de 2014 a 2016, del servicio de cirugía cardíaca. Se evaluaron las relaciones de variables operativas con la aparición de infección de sitio quirúrgico para determinar los factores significativos. Para la recolección de los datos se utilizaron los formularios de circulación extracorpórea y para el análisis las definiciones establecidas por Agencia Nacional de Vigilancia Sanitaria. Resultados: $66,2 \%$ eran del sexo masculine; $62,1 \%$ blancos; $47,3 \%$ hipertensos; $22,8 \%$ diabéticos y $91,7 \%$ cirugías electivas. Fiebre, edema local en 24 horas, dolor, necrosis en lesión operatoria y reintervención quirúrgica son los principales factores para incidencia de infecciones relacionadas al tiempo medio de circulación extracorpórea y de cirugía. Conclusión: la infección de sitio quirúrgico en revascularizaciones está relacionada con el tiempo de cirugía y de circulación extracorpórea.

Descriptores: Circulación extracorporea; Cirugía torácica; Enfermería; Infección hospitalaria.

\section{INTRODUÇÃO}

No Brasil, estima-se que são realizadas 350 Revascularização do Miocárdio (RM) a cada 1.000 .000 habitantes por ano. ${ }^{1} \mathrm{~A}$ RM tem a finalidade de revascularizar o músculo cardíaco afetado pela obstrução total ou parcial de uma ou mais artérias coronarianas e prolongar o tempo de vida destes pacientes. ${ }^{2}$

As principais complicações que podem ocorrer após a realização da $\mathrm{RM}$, destacam-se as infecções de sítio cirúrgico, as quais são consideradas adversidades que aumentam tempo de internação, investimentos financeiros e podem causar comorbidades e óbitos. ${ }^{2-3}$ Pesquisa indica que o tempo de Circulação Extracorpórea (CEC), idade dos pacientes, características da técnica cirúrgica utilizada na $\mathrm{RM}$ e antecedentes de saúde estão relacionadas ao desencadeamento desta complicação. ${ }^{4}$
Dentre as principais causas da infecção de sítio cirúrgico, destaca-se as ocasionadas pela CEC, a qual ocorre em cerca de $90 \%$ das cirurgias e é responsável por problemas pósoperatórias graves, ${ }^{5}$ como as de origem hidroeletrolítica, acidobásica, glicêmica, cardíaca, pulmonar, renal, neurológica, infecciosa e hepática. A literatura tem demonstra fragilidade na motivação para o elevado número de complicações com a técnica de CEC, no entanto, é nítido que o procedimento apenas simula a fisiologia, tornando, a técnica propícia a alterações. ${ }^{3}$

Outros fatores podem contribuir para o aparecimento de infecção de sítio cirúrgico, como a idade, gênero e tipo de cirurgia, cujas consequências são diretamente proporcionais ao tempo dispensado para diagnostico e devido tratamento, podendo ir de uma febre a o óbito. ${ }^{6}$ 


\section{JOURNAIOF

Necessitando-se, portanto, do aporte de medias apoiadas em evidências científicas, capazes de melhorar ações preventivas e de controle a este tipo de infecção. ${ }^{6}$ Diante da problemática apresentada é que emerge o objetivo deste estudo analisar a incidência de infecção do sítio cirúrgico em revascularizações do miocárdio.

\section{MATERIAIS E MÉTODOS}

Considerando a especificidade do objetivo proposto, esta pesquisa se qualifica como sendo de abordagem quantitativa, de modelo não experimental, retrospectivo, documental, descritivo e de corte transversal. $^{7}$

A coleta de dados foi realizada no período de abril a setembro de 2017 em um hospital de ensino de caráter público, caracterizado como de grande porte e referência em atendimento na região do Oeste do estado de São Paulo.

Foram analisados os dados secundários, oriundos de prontuários de pacientes submetidos à RM entre janeiro de 2014 e dezembro de 2016, em um período de três anos da implementação do serviço de cirurgia cardíaca. Foram selecionados, os prontuários com os seguintes critérios de inclusão: Apresentar descrição dos períodos transoperatórios de RM por meio da ficha de circulação extracorpórea do serviço de cirurgia cardíaca no período descrito. Foram excluídos os que não estavam disponíveis no serviço de arquivamento hospitalar.
No serviço em questão existem registros institucionais de $406 \mathrm{RM}$ realizadas no período de estudo, foram selecionados 314 para esta pesquisa, pois 92 prontuários encontravam-se como arquivo morto, tornando-se inacessíveis.

Utilizou-se as definições estabelecidas pela Agência Nacional de Vigilância Sanitária para identificar os fatores relacionados à infecção de sítio cirúrgico, tais como: febre, dor, hiperemia, calor local e calafrios, exames laboratoriais, secreção purulenta, abscesso, cultura de ferida operatória positiva, bem como registro de diagnóstico nosológico de infecção de sítio cirúrgico em prontuário. ${ }^{8}$

Para o desenvolvimento do banco de dados, armazenamento e tratamento estatístico das variáveis do estudo, utilizou-se o software $R$ versão 3.1.2® que é disponibilizado gratuitamente. ${ }^{9}$ Houve análise de variáveis nominais, categóricas e numéricas, disponíveis na ficha de circulação extracorpórea do serviço de cirurgia cardíaca e no prontuário.

Para verificação das variáveis de estudo foi utilizado o teste de teste $T$ student para verificação estatística das relações entre infecção de sítio cirúrgico e fatores determinantes. ${ }^{7}$ Foram considerados estatisticamente significantes os valores de $\mathrm{p}$ menores que 0,05 .

O desenvolvimento foi aprovado pelo Comitê de Ética em Pesquisa da instituição de ensino de vinculação dos autores e recebendo o Certificado de Apresentação para Apreciação Ética $n^{\circ}$ 65023417.2.0000.5515. 


\section{$\mathrm{r}$ NURSING

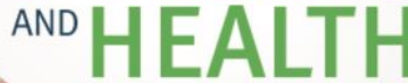 \\ RESULTADOS}

A amostra analisada foi composta de dados secundários de 314 RM. A análise dos dados para caracterização dos sujeitos quanto ao sexo, idade, etnia autodeclarada, doenças antecedentes e tipo de cirurgia realizada estão descritas na Tabela 1 .

Ao analisar a febre, edema perincisional e rubor no pós-operatório imediato além de dor, infecção tardia, uso de antibioticoterapia, necrose em ferida operatória, (re)abordagem operatória, cultura positiva e óbito relacionado ao procedimento,
ISSN 2236 - 1987

observou-se associação significativa com o tempo de CEC e/ou tempo de cirurgia, como apresentado na Tabela 2.

O tempo de CEC, o tempo de cirurgia e os diagnósticos de infecção de sitio cirúrgico foram submetidos ao cálculo do coeficiente da correlação de Person. A relação entre tempo de CEC e infecção de sitio cirúrgico foi estatisticamente significativa $(r=0,15)$, considera-se fraca, ou seja, quanto maior o tempo de CEC maior é o índice de infecção de sítio cirúrgico nas RM analisadas.

Tabela 1: Caracterização dos sujeitos de pesquisa $(n=314)$ quanto à sexo, idade, etnia autodeclarada, doenças antecedentes e tipo de cirurgia. Brasil, 2018.

\begin{tabular}{lcc}
\hline Variáveis & N & $\%$ \\
\hline Sexo & 208 & \\
$\quad$ Masculino & 106 & 66,2 \\
Feminino & 2 & 33,8 \\
Idade (anos) & 16 & 0,6 \\
$\quad$ 40 & 85 & 5,1 \\
$40-50$ & 100 & 27,0 \\
$51-60$ & 101 & 31,8 \\
$61-70$ & 10 & 32,3 \\
$71-80$ & & 3,2 \\
$>80$ & 195 & 62,3 \\
Cor declarada & 101 & 32,3 \\
Branco & 18 & 5,4 \\
Pardo & & \\
Preto & 270 & 47,3 \\
Doenças antecedentes (n=571) & 130 & 22,8 \\
Hipertensão Arterial Sistêmica & 113 & 19,8 \\
Diabetes Mellitus & 22 & 3,8 \\
Outros & 15 & 2,6 \\
Aparelho respiratório & 14 & 2,5 \\
Aparelho geniturinário & 7 & 1,2 \\
Nenhuma & & \\
Hormonal & & 91,7 \\
Tipo de Cirurgia & 288 & 8,3 \\
Eletiva & 26 & \\
Urgência & & \\
\hline
\end{tabular}


Tabela 2: Relação entre tempo de circulação extracorpórea e o tempo de cirurgia com febre, edema, rubor, dor, infecção tardia, antibioticoterapia no pós-operatório, necrose, (re)abordagem operatória, cultura positiva e óbito. Brasil, 2018.

\begin{tabular}{|c|c|c|c|c|c|c|}
\hline & & & $\begin{array}{c}\text { Média do Tempo de } \\
\text { CEC } \\
\text { (minutos) }\end{array}$ & & $\begin{array}{c}\text { Média do Tempo de } \\
\text { Cirurgia } \\
\text { (minutos) }\end{array}$ & \\
\hline Variáveis & $\mathrm{N}$ & $\%$ & & p-valor & & $\mathrm{p}$-valor \\
\hline Febre em 24h & & & & & & \\
\hline Sim & 16 & 5,0 & $111,6(\mathrm{Std}=40,53)$ & 0,025 & $222,6($ Std = 51,85) & \\
\hline Não & 297 & 95,0 & $78,9(\mathrm{Std}=30,67)$ & & $220,6(\mathrm{Std}=53,49)$ & 0,080 \\
\hline Edema local er & & & & & & \\
\hline Sim & 1 & 0,3 & $70,0(\mathrm{Std}=32,04)$ & 0,087 & $170,0($ Std $=53,21)$ & 0,031 \\
\hline Não & 313 & 99,7 & $80,6($ Std $=31,30)$ & & $220,9(\mathrm{Std}=60,87)$ & \\
\hline Rubor em 24h & & & & & & \\
\hline Sim & 1 & 0,3 & $88,0(\mathrm{Std}=32,04)$ & 0,081 & $195,0(\mathrm{Std}=53,27)$ & 0,062 \\
\hline Não & 313 & 99,7 & $80,5(\mathrm{Std}=31,30)$ & & $220,9(\mathrm{Std}=50,67)$ & \\
\hline Dor & & & & & & \\
\hline Sim & 156 & 49,6 & $78,8(\mathrm{Std}=28,85)$ & 0,034 & $222,1(\mathrm{Std}=51,85)$ & 0,092 \\
\hline Não & 157 & 50,4 & $82,3(\mathrm{Std}=31,30)$ & & $219,4(\mathrm{Std}=54,69)$ & \\
\hline Infecção de Sit & gico t & rdia & & & & \\
\hline Sim & 48 & 15,2 & $79,6($ Std $=27,97)$ & 0,090 & $224,6(\mathrm{Std}=49,23)$ & 0,072 \\
\hline Não & 265 & 84,8 & $80,7(\mathrm{Std}=32,71)$ & & $220,9(\mathrm{Std}=53,95)$ & \\
\hline Antibiótico no & eratór & media & & & & \\
\hline Sim & 310 & 98,7 & $80,6(S t d=32,08)$ & 0,062 & $220,7(\mathrm{Std}=53,16)$ & 0,056 \\
\hline Não & 4 & 1,3 & $80,0(\mathrm{Std}=26,45)$ & & $230,0(\mathrm{Std}=69,46)$ & \\
\hline Necrose em les & ratóri & & & & & \\
\hline Sim & 4 & 1,3 & $88,0($ Std $=15,87)$ & 0,064 & $240,0(\mathrm{Std}=39,05)$ & 0,047 \\
\hline Não & 310 & 98,7 & $80,8(\mathrm{Std}=32,11)$ & & $220,6(\mathrm{Std}=53,34)$ & \\
\hline (Re)abordagem & tória & & & & & \\
\hline Sim & 11 & 3,5 & $80,7(\mathrm{Std}=26,20)$ & 0,042 & $202,2(\mathrm{Std}=44,40)$ & 0,024 \\
\hline Não & 303 & 96,5 & $75,1(\mathrm{Std}=32,20)$ & & $221,4(\mathrm{Std}=53,44)$ & \\
\hline Cultura positiv & & & & & & \\
\hline Sim & 31 & 9,8 & $30,1(S t d=30,16)$ & 0,091 & $212,7(\mathrm{Std}=54,32)$ & 0,067 \\
\hline Não & 282 & 90,2 & $32,1(\mathrm{Std}=32,13)$ & & $221,6(\mathrm{Std}=53,11)$ & \\
\hline Sim & 21 & 0,0 & $90,0(\mathrm{Std}=31,54)$ & 0,085 & $239,0(\mathrm{Std}=57,51)$ & 0,066 \\
\hline Não & 292 & 93,4 & $79,9(\mathrm{Std}=31,97)$ & & $219,4(\mathrm{Std}=52,74)$ & \\
\hline
\end{tabular}

$\mathrm{N}$ - Número de prontuários avaliados; \% - Porcentagem; CEC - Circulação Extra-Corpórea; $\mathrm{p}$ - nível de significância.

\section{DISCUSSÃO}

A análise dos dados revela que $208(66,2 \%)$ pacientes eram do sexo masculino. Este resultado é esperado, pois existe prevalência deste gênero em complicações cardíacas e circulatórias. Isso semelhantemente também foi encontrado em um estudo realizado com 1.322 pacientes revascularizados para avaliação do risco de mediastinite identificou que $72,6 \%$ eram do gênero masculino. ${ }^{10}$

A maioria dos pacientes $(58,8 \%)$ tinham idade entre os 61 a 80 anos, dado semelhante a outro estudo, no qual encontraram média de idade de 62,4 anos com desvio padrão de 9,8 anos. ${ }^{10}$ Este fato é justificado por serem pacientes acometidos por doenças crônicas como diabetes e hipertensão arterial sistêmica, fatores 


\section{JOURNALOF \\ NURSING \\ ANO HEALTH}

de risco para o comprometimento cardiovascular, comum nestes pacientes. ${ }^{11}$

Diante da etnia predominante deste estudo, é importante enfatizar que no Brasil a miscigenação associada ao fator de autodeclararão étnica proporciona dados de prevalência de doenças do aparelho cardiovascular entre brancos. ${ }^{10-11} 0$ mesmo pode ser observado nesta pesquisa, na qual $62,1 \%$ autodeclaram-se brancos.

Quanto as doenças prévias à revascularização, houve nítido predomínio de Hipertensão Arterial Sistêmica (47,3\%) e Diabetes Mellitus $(22,8 \%)$, dados também identificados em outro estudo com o mesmo perfil de sujeitos. ${ }^{10}$ Isso se deve ao fato de serem doenças crônicas que podem provocar comorbidades, com início silencioso, tratamento dispendioso e sem melhora definitiva. ${ }^{11-12}$

As cirurgias foram realizadas de maneira eletiva em $91,7 \%$, tendo em vista tratar-se de um procedimento cirúrgico de elevado risco que necessita de adequado preparo humano, de materiais e estratégia institucional, sendo concebível realizar-se menos operações em caráter de urgência. ${ }^{13}$

O sintoma dor foi identificado em $49,6 \%$, corroborando resultados que evidenciaram que a CEC induz o extravasamento capilar difuso através da ativação das cininas e dos sistemas de complemento, contudo, essas induções resultam em edema intersticial importante, volume intravascular diminuído e volume corporal aumentado, ocasionando
ISSN 2236 - 1987

assim, a dor por processo inflamatório pós-traumático. ${ }^{14}$

Houve ainda, significância estatística na relação entre febre e tempo de CEC, sendo o sinal de febre relacionada ao trauma cirúrgico. Habitualmente, as causas infecciosas não se manifestam precocemente. As cirurgias cardíacas com CEC tendem apresentar mais febre, pois, a passagem de sangue por um circuito artificial na CEC impõe ao organismo um número importante de alterações, como a mudança do fluxo sanguíneo, aumento do gradiente de temperatura, estresse mecânico sobre os elementos do sangue devido ao contato com superfícies não endoteliais, filtros, compressão e turbulências, entre outros. Esse processo pode desencadear reações inflamatórias com deletérias consequências póscirúrgicas, em menor ou maior grau, sendo mais evidentes e prejudiciais em idosos, podendo se manifestar propriamente pela febre ocasionando até mesmo óbito. ${ }^{14-15}$

Os dados oriundos de 314 pacientes submetidos ao procedimento cirúrgico e incluídos na presente investigação, 21 (6,6\%) foram a óbito. Este resultado pode ser considerado muito bom em comparação com um estudo nacional incluindo cirurgias cardíacas que apresentou $38,9 \%$ óbitos em sua amostra. ${ }^{16}$

As taxas de infecção de sítio cirúrgico entre pacientes submetidos a cirurgias cardíacas são variáveis, com evidências de apenas $2 \%$ em outro estudo, ${ }^{17}$ podendo observar neste, valores baixos, com discreta semelhança, apontando 15,2\%. 


\section{JOURNALOF \\ NURSING \\ ${ }^{\text {ANO HEALTH }}$}

ISSN 2236 - 198

Uma das medidas sugeridas para a profilaxia da infecção de sítio cirúrgico é a antibioticoprofilaxia préoperatória, ${ }^{23}$ devendo ser realizada antes do procedimento, preferencialmente, utilizando cefalosporinas de primeira ou segunda geração. A implantação de protocolos de antiobioticoprofilaxia de maneira sistemática pela instituição hospitalar previne a ocorrência de infecção de sítio cirúrgico, reduzindo a carga microbiana presente no momento da cirurgia. A implementação dessas drogas deve ser baseada em evidências científicas, para garantir sucesso e diminuir os riscos de aumentar a resistência bacteriana, por meio da seleção de microorganismos. ${ }^{24}$

Contudo, a Organização Mundial da Saúde (OMS) apresenta algumas recomendações acerca do assunto, como o tempo certo para a profilaxia antibiótica cirúrgica pré-operatória, que se refere à prevenção de complicações infecciosas através da administração de um agente antimicrobiano eficaz antes da exposição à contaminação durante a cirurgia. ${ }^{25-26}$ Dentre elas, a administração de antibióticos antes da incisão cirúrgica, com forte recomendação, sendo realizado dentro de 120 minutos e não mais que isso, antes da incisão, considerando a meiavida do antibiótico e, mostra ainda, que a administração de antibióticos após a incisão cirúrgica provoca danos com aumento significativo do risco de infecção de sítio cirúrgico em comparação com a administração antes da incisão. ${ }^{21}$

Outra evidência mostra que uma concentração baixa de antibióticos nos 


\section{IOURNAIOF

tecidos no momento do fechamento da ferida está associada a maiores taxas de infecção de sítio cirúrgico. ${ }^{27}$

A OMS contraindica 0 prolongamento da administração de antibióticos após a conclusão do procedimento cirúrgico com o objetivo de prevenir infecção de sítio cirúrgico, ${ }^{21}$ o que torna possível relacionar o índice de infecção desta amostra com o fato do hospital onde a pesquisa foi realizada utilizar essa pratica.

Contudo, há evidência de qualidade moderada a partir de um número elevado de ensaios clínicos randomizados, 44 estudos incluídos em meta-análise global, ${ }^{21}$ mostrando que a administração de antibióticos prolongada no pós-operatório não tem nenhum benefício na redução da infecção de sítio cirúrgico após a cirurgia, quando comparados com a administração pré-operatória. ${ }^{28}$

No entanto, há evidências de que uma administração pós-operatória prolongada de antibióticos pode ser benéfica para reduzir 0 risco de infecção de sítio cirúrgico em cirurgias cardíacas, quando comparado a profilaxia pré-operatória. Considerando esta evidência de qualidade limitada e baixa a muito baixa no suporte de administração prolongada de antibióticos, acima mencionados, o Grupo de Desenvolvimento de Diretrizes da OMS decidiu posicionar-se contra 0 prolongamento da administração de antibióticos pós-operatório com a finalidade de prevenir a infecção de sítio cirúrgico. ${ }^{21,28}$
Cabe elucidar que a limitação deste estudo está relacionada a impossibilidade de analisar as técnicas operatórias empregadas em cada RM, haja vista se tratar de um estudo desenvolvido com dados secundários, retrospectivo. Todavia, sua relevância está na apresentação de dados que comprovaram estatisticamente a relação do tempo de CEC e do tempo de cirurgia com sinais de inflamação no sítio cirúrgico, tendo em vista que a infecção de sítio cirúrgico está relacionada à assistência à saúde e constitui uma intercorrência grave em pacientes submetidos à revascularização do miocárdio.

Apresenta-se como contribuições para a prática os conhecimentos apontados neste estudo que possibilitam evidências para a fundamentação de ações de controle específicas para minimizar a incidência da infecção do sítio cirúrgico em RM.

\section{CONCLUSÃO}

Os principais fatores relacionados com a incidência de infecção do sítio cirúrgico em revascularizações do miocárdio, baseado em dados secundários foram o tempo de CEC e o tempo de cirurgia, relacionados com febre, edema local em 24 horas, dor, necrose em lesão operatória, e a (re)abordagem operatória. Estes se apresentam com grande correlação ao surgimento das infecções de sítio cirúrgico, visto que são sinais e sintomas antecedentes o diagnóstico de infecção.

Considera-se que estes resultados podem contribuem para maior atenção ao paciente submetido à cirurgia cardíaca dada sua relevância, o risco 


\section{NURSING \\ AND \\ HEALTH}

de mortalidade e o custo das complicações. Além disso, podem para que o trabalho interprofissional possa ser planejado, baseando-se em práticas preventivas, bem como na monitorização dos fatores de risco.

\section{REFERÊNCIAS}

1 Gomes WJ, Mendonça JT, Braile DM, Domingo $M$. Cardiovascular surgery outcomes oportunity to rediscuss medical and cardiological care in the brazilian public health system. Rev bras cir cardiovasc [Internet]. 2007 [cited 2018 Nov 18];22(4). Available from:

http: / / www.scielo.br/scielo.php?scrip $\mathrm{t}$ =sci_arttext\&pid=S0102-

\section{2}

2 Piegas LS, Timerman A, Feitosa GS, Nicolau JC, Mattos LAP, Andrade MD et al. $V$ diretriz da sociedade brasileira de cardiologia sobre tratamento do infarto agudo do miocárdio com supra desnível do segmento ST. Arq bras cardiol [Internet]. 2015 ago[acesso em 2018 nov 10];105(2):1-121. Disponível em:

http: / / publicacoes.cardiol.br/2014/di retrizes/2015/02_TRATAMENTO\%20DO \%20IAM\%20COM\%20SUPRADESNIVEL\%20 DO\%20SEGMENTO\%20ST.pdf

3 Oliveira JMA, Silva AMF, Cardoso SB, Lima FF, Zierer MS, Carvalho ML. Complicações no pós-operatório de cirurgia cardiovascular com circulação extracorpórea. Rev interdisciplinar [Internet]. 2015[acesso em 2018 nov 10];8(1):9-15. Disponível em: https://revistainterdisciplinar.uninova fapi.edu.br/index.php/revinter/articl e/view/584
ISSN $2236-1987$

4 Gelape CL. Surgical Wound Infection Following Heart Surgery. Arq bras cardiol [Internet]. 2007[cited 2018 Nov 10];89(1):e3-e9.

Available from: http://www.scielo.br/pdf/abc/v89n1 /en_13.pdf

5 Cardoso SB. Circulação extracorpórea e cirurgia cardiovascular. Sociedade de cardiologia do estado do Piauí [Internet]. 2012[acesso em 2018 nov 21]. Disponível em: http://sociedades.cardiol.br/pi/notici as/artigo-circulacao.pdf

6 Reis RG, Rodrigues MCS. Infecção de sítio cirúrgico pós-alta: ocorrência e caracterização de egressos de cirurgia geral. Cogitare enferm [Internet]. 2017 [acesso em 2018 nov 18];22(4):1-14. Disponível em: https://revistas.ufpr.br/cogitare/arti cle/view/51678/pdf

7 Denise F, Polit CT, Beck P. Nursing research: generating and assessing evidence for nursing practice. $10^{\mathrm{a}}$ ed. Philadelphia: Wolters Kluwer Health; 2017

8 Ministério da Saúde (BR). Agência nacional de vigilância sanitária. Critérios Diagnósticos de Infecção Relacionada à assistência à saúde [Internet]. Brasília: ANVISA; 2017[acesso em 2017 abr 19]. Disponível em: http://portal.anvisa.gov.br/document s/33852/3507912/Caderno+2+-

+Critérios+Diagnósticos+de+Infecção+

Relacionada+à+Assistência+à+Saúde/7 485b45a-074f-4b34-8868-

$61 \mathrm{f} 1 \mathrm{e} 5724501$

9 R Foundation. The R Project for Statistical Computing [Internet]. 2017 [acesso em 27 de maio de 2017]. 


\section{JOURNALOF \\ NURSING \\ AND \\ Disponível em: https://www.r- project.org/}

ISSN $2236-1987$

10 Oliveira FS, Freitas LDO, RabeloSilva ER, Costa LM, Kalil RAK, Moraes MAP. Predictors of mediastinitis risk after coronary artery bypass surgery: applicability of score in 1.322 cases. Arq bras cardiol [Internet]. 2017[cited 2018 Nov 10];109(3):207-12. Available from:

http: / /www.scielo.br/scielo.php?pid= S0066782X2017000900207\&script=sci_ arttext\&tlng=pt

11 Pallo PAO, Levy-Neto $M$, Pereira RMR, Shinjo SK. Relapsing polychondritis: prevalence of cardiovascular diseases and its risk factors, and general disease features according to gender. Rev bras reumatol [Internet]. 2017 [cited 2018 Nov 10];57(4):338-45. Available from: https: / / reader.elsevier.com/reader/s $\mathrm{d} / \mathrm{pii} / \mathrm{S} 0482500417300438$ ?token=B12B 6A95FC582C4B8832881864845B89525D 7881D91A83C28A392850EC44A3C926C3 D31086CE8F6AB663FC7C23A4D5A3

12 de Araújo HV, Soares EA. Quality of life of patients who undergone myocardial revascularization surgery. Rev bras enferm [Internet]. 2017[cited 2018 Nov 10];70(2):273-81. Available from:

http: / /www.scielo.br/pdf/reben/v70 n2/pt_0034-7167-reben-70-020257.pdf

13 Madeira S, Raposo L, Brito J, Rodrigues R, Gonçalves P, Teles R, et al. Potential utility of the SYNTAX score 2 in patients undergoing left main angioplasty. Arq bras cardiol [Internet] 2016[cited 2018 Nov 10];106(4):270-8. Available from: http: / /www.scielo.br/pdf/abc/v106n 4/0066-782X-abc-20160038.pdf

14 Dessotte CAM, Rodrigues HF, Furuya RK, Rossi LA, Dantas RAS. Stressors perceived by patients in the immediate postoperative of cardiac surgery. Rev bras enferm[Internet] 2016. [cited 2018 Nov 10];69(4):741-50. Available from:

http://www.scielo.br/pdf/reben/v69 n4/en_0034-7167-reben-69-040741.pdf

15 Rhee C, Sax PE. Evaluation off ever and infections in cardiac surgery patients. Semin cardiothorac vasc anesth [Internet]. 2015[cited 2018 Nov 10];19(2):143-53 Available from: https: / /journals.sagepub.com/doi/ab s/10.1177/1089253214538524

16 Rodrigues JASN, Ferreti-Rebustini REL, Poveda VB. Infecção do sítio cirúrgico em pacientes submetidos a transplante cardíaco. Rev. latinoam enferm (online) [Internet]. 2016 jul/ago[acesso em 2018 nov 10];24:18; Acesso em: http://www.scielo.br/pdf/rlae/v24/p t_0104-1169-rlae-24-02700.pdf

17 Ho JK, Sung-Ho J, Jae JK, Joon BK, Suk JC, Tae-Jin Y, et al. Early postoperative complications after heart transplantation in adult recipients: asan medical center experience. Korean j thorac cardiovasc surg [Internet] 2016.[cited 2018 Nov 10];46(6):426-32. Available from: https://pdfs.semanticscholar.org /a9bc/399db85b86f7a12b8dcc65bbdb7 49822ae76.pdf

18 Center for Disease Control (CDC). $\mathrm{CDC} / \mathrm{NHSN}$ surveillance definitions for specific types of infections [Internet]. 2015[cited 2018 Nov 10]. Available 
from:

https://www.cdc.gov/nhsn/pdfs/psc manual/17pscnosinfdef_current.pdf

19 Lemos FN, Oliveira AV, De Sousa MG. Infecção de sítio cirúrgico: estudo prospectivo de 2.149 pacientes operados. Rev col bras cir [Internet]. 2018[acesso em 2018 nov 10];26(2):109-13. Disponível em: http://www.scielo.br/pdf/rcbc/v26n2 /07.pdf

20 Rocha JPJ, Lages CAS. O enfermeiro e a prevenção das infecções do sítio cirúrigico. Cadernos unifoa [Internet]. 2016 abr[acesso em 2018 nov 21];11(30):117-28. Disponível em: http://revistas.unifoa.edu.br/index.p $\mathrm{hp} /$ cadernos/article/view/357/440

21 World Health Organization (WHO). Global guidelines for the prevention of surgical site infection [Internet]. Geneva: World Health Organization; 2016[cited 2018 Nov 21]. Available from:

http:/ / apps.who.int/iris/bitstream/h andle/10665/250680/9789241549882eng.pdf;jsessionid=9D42DCF17F76F697 DC5C9E871E3430A6? sequence $=1$

22 Borges, SO. Risco de recuperação cirúrgica retardada [Trabalho de Conclusão de Curso]. Rio de Janeiro (RJ): Universidade Federal Fluminense; 2016.

23 Finkelstein R, Rabino G, Mashiach T, Bar-El Y, Adler Z, Kertzman V. Effect of preoperative antibiotic prophylaxis on surgical site infections complicating cardiac surgery. Infect control hosp epidemiol [Internet]. 2014[cited 2018 Nov 10];35(1):69-74. Available from: https://www.ncbi.nlm.nih.gov/pubm ed/24334801
24 Najjar PA, Smink DS. Prophylactic antibiotics and prevention of surgical site infections. Surgical clinics of north america [Internet]. 2015[cited 2018 Nov 10];95(2):269-83. Available from: https://linkinghub.elsevier.com/retri eve/pii/S0039-6109(14)00213-8

25 Gualandro DM, Yu PC, Calderaro D, Marques AC, Pinho C, Caramelli B, Casella IB. II guidelines for perioperative evaluation of the brazilian society of cardiology. Arq bras cardiol [Internet]. 2011[cited 2018 Nov 10];96(3suppl1):1-68. Available from: http://publicacoes.cardiol.br/consens o/2011/II_diretriz_perioperatoriaingles.pdf

26 Anderson DJ, Podgorny K, BerriosTorres SI, Bratzler DW, Dellinger PE, Greene G, et al. Strategies to prevent surgical site infections in acute care hospitals: 2014 update. Infect control hosp epidemiol [Internet]. 2014[cited 2018 Nov 21];35(6):605-27. Available from:

https://www.ncbi.nlm.nih.gov/pmc/a rticles/PMC4267723/

27 Gualandro DM, Yu PC, Caramelli B, Marques AC, Calderaro D, Fornari LS, et al. 3rd guideline for perioperative cardiovascular evaluation of the brazilian society of cardiology. Arq bras cardiol [Internet]. 2017 Jan/Feb[cited $2018 \quad$ Nov 21];109(3suppl1):1-104. Available from:

https://www.ncbi.nlm.nih.gov/pmc/a rticles/PMC5629911/

28 Mackeen $A D$, Packard RE, Ota E, Berghella V, Baxter JK. Timing of intravenous prophylactic antibiotics for preventing postpartum infectious 


\section{JOURNALOF

morbidity in women undergoing cesarean delivery. Cochrane database syst rev [Internet]. 2014[cited 2018 Nov 10];12:51-6. Available from: https: / /www.cochranelibrary.com/cd sr/doi/10.1002/14651858.CD009516.p ub2/full

Data de submissão: $30 / 07 / 2018$

Data de aceite: 18/11/2018

Data de publicação: 29/11/2018 